\title{
Low-temperature electron-phonon heat transfer in metal films.
}

\author{
S. Cojocaru and D. V. Anghel \\ Horia Hulubei National Institute For Physics And \\ Nuclear Engineering, RO-07r125, Magurele, Romania
}

\begin{abstract}
We consider the deformation potential mechanism of the electron-phonon coupling in metal films and investigate the intensity of the associated heat transfer between the electron and phonon subsystems. The focus is on the temperature region below dimensional crossover $T<T^{*}$ where the thermally relevant vibrations are described in terms of a quasi-two-dimensional elastic medium, while electron excitations behave as a three-dimensional Fermi gas. We derive an explicit expression for the power $P(T)$ of the electron-phonon heat transfer which explains the behavior observed in some experiments including the case of metallic film supported by an insulating membrane with different acoustic properties. It is shown that at low temperatures the main contribution is due to the coupling with Lamb's dilatational and flexural acoustic modes.
\end{abstract}




\section{INTRODUCTION}

In modern electronic devices the nanoscale miniaturization and sub-Kelvin temperatures are quite common, however the physical phenomena taking place in such conditions are far from complete understanding and attract a great deal of research interest. In this work we address an aspect of the electron-phonon interaction in confined systems related to heat transfer between electrons and phonons. This is an open problem in the fundamental sense with a direct connection to current research activity and important applications in a variety of fields from nanoelectronics to astrophysics. For instance, in a recent paper [1] the

principle of electronic cooling [2] has been used to realize the "coolest microfridge" reaching a record temperature of less than $30 \mathrm{mK}$. In a typical setup a cooled metal part is suspended or mounted on an insulating support layer in contact with superconductors forming two symmetrically biased NIS (normal metal-insulator-superconductor) tunnel junctions. In this setup "hot" electrons from above the Fermi level are evacuated from the normal metal island, while "cold" electrons are injected below the Fermi level. Such microdevices can be mounted directly on a chip for cooling qubits or ultrasensitive low-temperature detectors, e.g., bolometers or calorimeters, where the biased NIS tunnel junctions can also be used for precision thermometry down to milli-Kelvin temperatures [3]. An important physical phenomenon controlling the cooling power is the heat transfer between electrons and phonons mediated by their coupling, $H_{e-p}$, when phonons are emitted and absorbed by electrons. When electrons are heated by an external source in a stationary regime one can assume their energy distribution to be characterized by a temperature $T_{e}$ while the distribution of phonons corresponds to some lower temperature $T_{p}$. In many situations the temperature gradients are sufficiently small so that we also assume that space variation of $T_{e}$ and $T_{p}$ can be neglected. When both subsystems are bulklike (three-dimensional) the rate $P$ at which electron energy is transferred to phonons has been obtained by considering the deformationpotential mechanism of electron-phonon coupling, which relates the local density fluctuation to the variation of the Fermi energy [4], and $P(T)$ has been shown to vary as $T^{5}$ at low temperatures (see, e.g., [5-7]):

$$
P=\Sigma V_{e l}\left(T_{e}^{5}-T_{p}^{5}\right)
$$

Here $V_{e l}$ is the volume of the metal and $\Sigma$ depends on the electron-phonon coupling and other properties of the sample. This form has been derived for simple metals in the case 
when disorder is not strong $(q l>>1$, where $q$ is the phonon wave vector and $l$ is the electron mean free path) and which is also assumed in the present work. It should be mentioned that for disordered films a form with a stronger $\left(T^{6}\right)$ low-temperature behavior has been found [8, 9]. The dependence in Eq. (11) was confirmed in many experimental situations and is a standard formula assumed for the analysis of experimental data, e.g., [1, 3]. However, the finite thickness of a film $L$ eliminates the possibility of longer waves to propagate in this direction. Consequently, when temperatures fall below the dimensional crossover threshold $T<T^{*} \simeq c \hbar /\left(k_{B} L\right)$, where $c$ is the sound velocity, the wavelength of the thermally relevant phonons becomes longer than $L$ and we may treat the phonon subsystem in terms of a confined elastic medium. Respectively, thermal properties, including the electron-phonon heat transfer, are dominated by the vibrational eigenmodes corresponding to such a quasitwo-dimensional geometry. For values of $L$ of the order of $100 \mathrm{~nm}$ and for sound velocities of the order of $10 \mathrm{~km} / \mathrm{s}$, the value of $T^{*}$ is of the order of $1 \mathrm{~K}$. Therefore size related effects in electron-phonon systems have become an important part of the physics at the nanoscale, e.g., [10, 11]. In a number of experimental studies it has been found that the temperature dependence is best represented by the $T^{x}$ with significantly lower values of $x$ [12 14]. On the other hand, a theoretical investigation of the surface effects for a halfspace geometry [15], including the surface specific Rayleigh phonon modes, has shown that the value of $x$ is actually larger than 5 and at sufficiently low temperatures it exceeds 6 . Nevertheless, the growth of the exponential $x$ with decreasing temperature has been later qualitatively confirmed in some experiments when metallic films were deposited on bulky substrates [13, 16]. For a quasi-one-dimensional geometry (metallic nanowire) the model has been studied theoretically in [17] where the $T^{3}$ analog of Eq. (11) has been obtained. Although in [18] it was argued that for $\mathrm{Al}$ nanowires with $65 \times 90$-nm cross-section a better fit is achieved with the standard exponential $x=5$, the results are still inconclusive since no dimensional crossover was observed around the anticipated temperature, $0.45 \mathrm{~K}$. In contrast, a clear indication of a quasi-two-dimensional crossover in electron-phonon dominated heat flow with a distinct power law $(x<4.5)$ has been reported in Refs. [12, 13]. Remarkably, the respective samples had also a strongly enhanced density of the heat flux compared to the thicker samples, which remained in the "bulk" regime and did not show a crossover behavior for the considered temperature interval. Typically, the metallic film is either deposited on an insulating membrane, Fig [1, or suspended on top of superconducting electrodes [1]. 


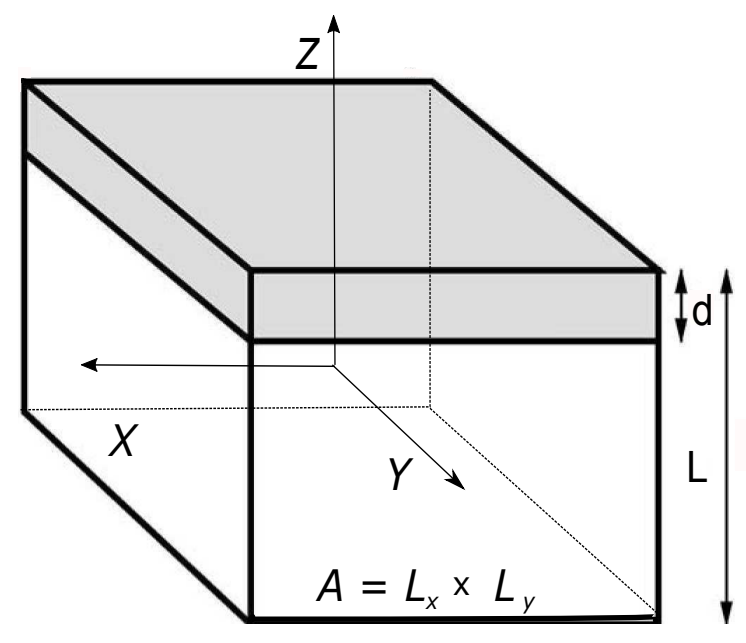

FIG. 1: Insulating membrane of thickness $L-d$ covered with a metal film (gray) of thickness $d$ and surface area $A$.

The phonon spectrum of a quasi-two-dimensional system is quite different from that of a bulk. Vibrational eigenstates of such a slablike structure can not be separated into longitudinal and transverse waves; instead in the elastic continuum approximation the spectrum is given by the Lamb eigenmodes (in addition to shear waves) actually representing a mixture of both [19]. The electron scattering by Lamb phonon modes has been studied earlier for semiconductor quantum wells (QWs) in [20], [10] and in a double heterostructure QW including the piezoelectric coupling in [21]. It has been shown that the scattering rate $\tau^{-1}$ is dominated by coupling to the flexural acoustic mode (e.g., $\tau^{-1} \sim T^{5 / 2}, T^{7 / 2}$ ) due to its characteristic quadratic dispersion and high density of states, in contrast to the "standard" linear dispersion of the, e.g., dilatational Lamb mode, which has a negligible contribution ( e.g., $\tau^{-1} \sim T^{6}$ ). In the present work the electron excitations in the metal film are treated as a three-dimensional Fermi gas interacting with the quasi-two-dimensional phonon subsystem. Thus, electrons are described by the parabolic dispersion with an effective electron mass $\epsilon_{\mathbf{k}}=\hbar^{2} \mathbf{k}^{2} / 2 m$ [notations for the components of the wave vector $\mathbf{k}=\left(\mathbf{k}_{\|}, k_{z}\right)$ correspond to Fig.1 and a plane-wave function $\Psi_{\mathbf{k}}(\mathbf{r}, \mathbf{t})=\exp (i \mathbf{k r}-i \epsilon t / \hbar) / \sqrt{V_{e}}=\psi\left(k_{z} ; z\right) \exp \left(i \mathbf{k}_{\|} \mathbf{r}_{\|}-i \epsilon t / \hbar\right) / \sqrt{A}$, where the electronic volume is $V_{e}=d A$ and $\psi\left(k_{z} ; z\right)$ is given in the next Section. We will use alternatively either the cylindrical coordinate system with $k_{z}$ normal to the film and the in-plane vector $\mathbf{k}_{||}$at angle $\phi$, or the spherical system with the two angles denoted as $\theta$ and $\varphi$ and $k=|\mathbf{k}|$. It should 
be mentioned that in ultrathin metal films electron confinement can result in the formation of the quantum well state with $\psi\left(k_{z} ; z\right) \sim \sin \left(k_{z} z\right)$ and $k_{z}=n \pi / d$, where the quantum numbers $n=1,2,3 \ldots$ correspond to the electron quasi-two-dimensional energy sub-bands. However, in contrast to semiconductors, the QW state is more difficult to observe in metal films thicker than a few nm (e.g., [22]), because the electron de Broglie wavelength is comparable to the interatomic distance and signatures of size quantization are easily smeared out by film imperfections of the same length scale, e.g., surface roughness. Thus, the planewave form of $\psi\left(k_{z} ; z\right)$ is an approximation, which is meant to describe the situation of a not very thin film where electron band structure can still be viewed as bulklike, while the phonon spectrum is dominated by quasi-two-dimensional modes. An important distinctive feature of this situation is that, although the phonons propagate parallel to the plane of the film, they can nevertheless produce electron scattering with a change of momentum in the direction normal to the plane, i.e., $\hbar k_{z}^{\prime} \neq \hbar k_{z}$. This paradox is due to the displacement field pattern characteristic of the Lamb waves (see below) which allows electrons to couple both to longitudinal (in-plane) and transverse (out-of -plane) components of the vibrations (see also the discussion in [23] for the case of a nanowire). The effect pertains primarily to the flexural modes and leads to a non-trivial modification of the heat transfer.

In an often used experimental setup the metallic strip is in contact with an insulating support membrane which can modify both the phonon spectrum and the flow of the heat produced in the metal and transmitted through the boundaries (e.g., [24]). Thus, the boundary can give rise to interface guided Stoneley phonon modes[25, 26]. However, for a solid-solid boundary the conditions on the parameters of the media (densities and sound velocities) required for the existence of Stoneley modes are very restrictive (see, e.g., Ref. [27]). We return to this issue in the next chapter. The heat transfer can also depend on the coupling of phonons in the film to their own bath; e.g., in [16, 28] it has been found that the distribution of phonons available for interaction with electrons in metal films can remain relatively unaffected by the substrate. In general, due to a mismatch of properties on the interface between two materials the phonons will scatter and produce a thermal boundary resistance; the associated Kapitza heat flow then depends on the difference of phonon temperatures of the two materials and is usually given by $\kappa\left(T_{p, 1}^{4}-T_{p, 2}^{4}\right)$ [29, 30]. There are other effects that can contribute to the heat transport, e.g., related to the operational principle of electron microcoolers, when there exists a heat backflow from the superconductor to the metal island 
[31].

Below we will consider the heat flux derived from the electron-phonon coupling for the structure shown in Fig 1 by first assuming homogeneous elastic properties of the compound slab of volume $V_{p}=L \times A$ [21], its total mass $M$, and mass density $\rho=M / V_{p}$. The case of a suspended metallic film corresponds to the condition $L=d$. To account for the modification of the phonon spectrum when the metal film is deposited on the insulating membrane with acoustic characteristics different from the metal, one can consider different models of their bonding (see, e.g., [32]), however we assume that the contact between the two media is rigid. It should be stressed that knowledge of the phonon spectrum is not sufficient when considering the coupling to electrons and one should also determine the properly normalized amplitudes of the phonon field.

\section{ELECTRON-PHONON COUPLING AT LOW TEMPERATURES}

We define the rectangular coordinate system in such a way that $z= \pm L / 2$ corresponds to the top and bottom surfaces of the slab. Elastic vibrations are described by the vector field of relative displacements $\mathbf{u}=\mathbf{u}(\mathbf{r})$ [19] expanded in the series of quantized eigenmodes of the continuum elasticity equation for the vibrations of a rectangular plate [20]:

$$
\mathbf{u}(\mathbf{r})=\sum_{\eta, \mathbf{q} \|} \sqrt{\frac{\hbar}{2 \rho A \omega_{\eta}}}\left[a_{\eta}\left(\mathbf{q}_{\|}\right)+a_{\eta}^{+}\left(-\mathbf{q}_{\|}\right)\right] \mathbf{w}_{\eta}\left(\mathbf{q}_{\|}, z\right) \exp \left(i \mathbf{q}_{\|} \mathbf{r}_{\|}\right) .
$$

Here $a_{\eta}^{+}\left(\mathbf{q}_{\|}\right)$and $a_{\eta}\left(\mathbf{q}_{\|}\right)$are phonon creation and annihilation operators, and $\omega_{\eta}$ is the set of normal vibration frequencies corresponding to the branches $(\xi)$ of the three types $(\alpha)$ of eigenmodes $\eta=(\alpha=\{h, d, f\}, \xi=1,2, \ldots)$, where $h$ is horizontal shear, $d$ is the dilatational mode, and $f$ is the flexural mode. The quantum amplitudes $\mathbf{w}_{\eta}\left(\mathbf{q}_{\|}, z\right)$ are orthonormalized over the thickness $L$

$$
\int_{-L / 2}^{L / 2} \mathbf{w}_{\eta}\left(\mathbf{q}_{\|}, z\right)^{\dagger} \mathbf{w}_{\eta^{\prime}}\left(\mathbf{q}_{\|}, z\right) d z=\delta_{\eta, \eta^{\prime}}
$$

The deformation potential coupling (see, e.g., [4] ) $H_{e-p}=\frac{2}{3} E_{F} \int_{V_{e}} d^{3} \mathbf{r} \Psi^{\dagger}(\mathbf{r}) \Psi(\mathbf{r}) \nabla \cdot \mathbf{u}(\mathbf{r})$ (where $E_{F}=\hbar^{2} \mathbf{k}_{F}^{2} / 2 m$ is the Fermi energy; see also the discussion for $\mathrm{Cu}$ in [15]) is then determined by the divergence of the displacement vector and takes the following form in the second quantization:

$$
H_{e-p}=\sum_{\mathbf{k}_{\| \mid}, \mathbf{q}_{\|}, \eta, k_{z}, k_{z}^{\prime}}\left[g_{\eta, \mathbf{q}_{\|}}^{k_{z}, k_{z}^{\prime}} c_{\mathbf{k}_{||}^{\dagger}+\mathbf{q}_{\|}, k_{z}^{\prime}} c_{\mathbf{k}_{\|}, k_{z}} a_{\eta}\left(\mathbf{q}_{\|}\right)+\left(g_{\eta, \mathbf{q}_{\|}}^{k_{z}, k_{z}^{\prime}}\right)^{*} c_{\mathbf{k}_{\| \mid}^{\dagger}-\mathbf{q}_{\|}, k_{z}^{\prime}} c_{\mathbf{k}_{\|}, k_{z}} a_{\eta}^{\dagger}\left(\mathbf{q}_{\|}\right)\right] .
$$


Here $c_{\mathbf{k}_{||}, k_{z}}^{+}$and $c_{\mathbf{k}_{\|}, k_{z}}$ are the electron creation and annihilation operators; the electronphonon matrix elements are given by the expression:

$$
g_{\eta, \mathbf{q} \|}^{k_{\|}, k_{z}^{\prime}}=\frac{2}{3} E_{F} \sqrt{\frac{\hbar}{2 \rho A \omega_{\eta}}} \int_{L / 2-d}^{L / 2} \psi^{*}\left(k_{z}^{\prime} ; z\right) \psi\left(k_{z} ; z\right)\left(i \mathbf{q}_{\|} \cdot \mathbf{w}_{\eta}\left(\mathbf{q}_{\|}, z\right)+\frac{\partial w_{\eta}^{z}\left(\mathbf{q}_{\|}, z\right)}{\partial z}\right) d z .
$$

Note that for the considered quasi-two-dimesnional geometry the momentum conservation rule works only for the in-plane components of the wave vectors, $\mathbf{k}_{\|}^{\prime}=\mathbf{k}_{\|} \pm \mathbf{q}_{\|}$, and the electron-phonon coupling allows scattering with the change of the $k_{z}$ component. The coordinate system is oriented in the plane as shown in Fig 1 so that we choose $x$ to correspond to the propagation direction of the wave $\mathbf{q}_{\|}=(q, 0,0)$. Then from the displacement patterns of the three eigenmodes, $\mathbf{w}_{h}(q, z)=\left(0, w_{h}^{y}, 0\right)$ and $\mathbf{w}_{d, f}(q, z)=\left(w_{d, f}^{x}, 0, w_{d, f}^{z}\right)$, one can easily see that only the $d$ and $f$ modes couple to electrons. We further the mode index whenever this does not cause confusion and then the amplitudes resulting from the solutions of the elasticity equations for the free surface boundary conditions can be represented as follows (see Refs. [33, 34]):

$$
\begin{aligned}
& w_{d}^{x}=i q_{t} F_{d}\left[2 q^{2} \cos \left(\frac{q_{t} L}{2}\right) \cos \left(q_{l} z\right)+\left(q_{t}^{2}-q^{2}\right) \cos \left(\frac{q_{l} L}{2}\right) \cos \left(z q_{t}\right)\right], \\
& w_{d}^{z}=q F_{d}\left[-2 q_{t} q_{l} \cos \left(\frac{q_{t} L}{2}\right) \sin \left(q_{l} z\right)+\left(q_{t}^{2}-q^{2}\right) \cos \left(\frac{q_{l} L}{2}\right) \sin \left(z q_{t}\right)\right] .
\end{aligned}
$$

and

$$
\begin{aligned}
& w_{f}^{x}=i q_{t} F_{f}\left[2 q^{2} \sin \left(\frac{q_{t} L}{2}\right) \sin \left(q_{l} z\right)+\left(q_{t}^{2}-q^{2}\right) \sin \left(\frac{q_{l} L}{2}\right) \sin \left(z q_{t}\right)\right], \\
& w_{f}^{z}=q F_{f}\left[2 q_{t} q_{l} \sin \left(\frac{q_{t} L}{2}\right) \cos \left(q_{l} z\right)-\left(q_{t}^{2}-q^{2}\right) \sin \left(\frac{q_{l} L}{2}\right) \cos \left(z q_{t}\right)\right] .
\end{aligned}
$$

The multipliers $F_{d}$ and $F_{f}$ are determined by the normalization condition (3). The above expressions are equivalent to Eqs. (10) - (11) and (15) - (16) obtained in [20], as can be easily checked by taking into account that the auxiliary parameters $\left(q_{t}, q_{l}\right)$ satisfy the eigen-frequency equations:

$$
\frac{-4 q^{2} q_{l} q_{t}}{\left(q^{2}-q_{t}^{2}\right)^{2}}=\frac{\tan \left(q_{t} L / 2\right)}{\tan \left(q_{l} L / 2\right)}
$$

for the dilatational mode, and

$$
\frac{-4 q^{2} q_{l} q_{t}}{\left(q^{2}-q_{t}^{2}\right)^{2}}=\frac{\tan \left(q_{l} L / 2\right)}{\tan \left(q_{t} L / 2\right)}
$$


for the flexural mode. The closure equation is secured by the "Snell law":

$$
\omega=c_{l} \sqrt{q_{l}^{2}+q^{2}}=c_{t} \sqrt{q_{t}^{2}+q^{2}},
$$

where $c_{l, t}$ are the longitudinal and transverse sound velocities of the material with the Lame coefficients $\lambda$ and $\mu$ and mass density $\rho$ :

$$
c_{t}=\frac{\mu}{\rho} ; c_{l}=\frac{\lambda+2 \mu}{\rho}: \quad J \equiv c_{t}^{2} / c_{l}^{2}<1 / 2 .
$$

From the analysis of the solutions, Refs. [20] and [34], it follows that to obtain an explicit expression for the leading low-temperature terms of $P$ it is sufficient to consider the lowestenergy part of the phonon spectra (i.e., acoustical waves), so that the branch index $\xi=1$ can now be dropped, $\omega_{\eta}(q)=\omega_{\alpha=d, f}$. Then auxiliary parameters for the $f$ mode are both purely imaginary $\left(q_{t . l}=i p_{t, l}\right)$, while for the $d$ wave $q_{t}$ is real and $q_{l}=i p_{l}$ is imaginary. After lengthy but straightforward calculations we obtain the explicit form of the solution of the above equations in the long-wavelength approximation:

$$
\begin{gathered}
\omega_{d} \simeq 2 q c_{t} \sqrt{1-J}, F_{d}^{-2} \simeq 16 q^{6}(3-4 J)(1-J)^{2} L, \quad q_{t}^{d} \simeq q \sqrt{3-4 J}, \quad p_{l}^{d} \simeq q(1-2 J) . \\
\omega_{f} \simeq q^{2} L c_{t} \sqrt{(1-J) / 3}, F_{f}^{-2} \simeq\left(q^{2} L\right)^{6} L(1-J)^{2} / 36 \\
p_{t}^{f} \simeq-q+q^{3} L^{2}(1-J) / 6, \quad p_{l}^{f} \simeq-q+q^{3} L^{2} J(1-J) / 6 .
\end{gathered}
$$

These expressions can now be used for the calculation of the electron-phonon matrix elements (5]):

$$
\left|g_{\alpha, \mathbf{q} \|}^{k_{z}, k_{z}^{\prime}}\right|^{2}=\left|F_{\alpha}\right|^{2}\left[q_{t} q\left(q_{l}^{2}+q^{2}\right)\right]^{2} \frac{8 \hbar E_{F}^{2}}{9 \rho A \omega_{\alpha}}\left\{\begin{array}{c}
\cos ^{2}\left(L q_{t} / 2\right), \alpha=d \\
\sin ^{2}\left(L q_{t} / 2\right), \alpha=f
\end{array}\right\} S\left(k_{z}, k_{z}^{\prime}, \alpha, q_{l}\right)
$$

where the overlap integral $S\left(k_{z}, k_{z}^{\prime}, \alpha, q_{l}=i p_{l}\right)$ is

$$
S\left(k_{z}, k_{z}^{\prime}, \alpha, q_{l}=i p_{l}\right)=\left|\int_{L / 2-d}^{L / 2} \psi^{*}\left(k_{z}^{\prime} ; z\right) \psi\left(k_{z} ; z\right)\left\{\begin{array}{l}
\cosh \left(z p_{l}\right), \alpha=d \\
\sinh \left(z p_{l}\right), \alpha=f
\end{array}\right\} d z\right|^{2} .
$$

As we have already mentioned, the scattering processes described by the Hamiltonian (4) do not require conservation of the $z$ component of the electron wave vector. However, in the long wave limit the overlap integrals can be approximated by taking $\cosh \left(z p_{l}\right) \simeq 1$ and $\sinh \left(z p_{l}\right) \simeq z p_{l}$ in (14) and using the orthonormality of the $\psi\left(k_{z} ; z\right)$. The respective expressions for the $d$ and $f$ modes simplify to:

$$
S\left(k_{z}, k_{z}^{\prime}, d, q_{l}=i p_{l}\right)=\delta_{k_{z}, k_{z}^{\prime}}
$$


and

$$
S\left(k_{z}, k_{z}^{\prime}, f, q_{l}=i p_{l}\right)=\left|p_{l}\right|^{2}\left|\int_{L / 2-d}^{L / 2} z \psi^{*}\left(k_{z}^{\prime} ; z\right) \psi\left(k_{z} ; z\right) d z\right|^{2} .
$$

Thus, in the long-wavelength approximation the interaction with dilatational modes effectively preserves the electron momentum $k_{z}$, unlike the interaction with flexural modes. As discussed in the Introduction, we assume the plane-wave expression for the $\psi\left(k_{z} ; z\right)$ function:

$$
\psi\left(k_{z} ; z\right)=\sqrt{\frac{1}{d}} \exp \left(i k_{z}(z+d-L / 2)\right)
$$

This implies, as pointed out in Ref. [23] for the case of a nanowire, that the electron-phonon coupling containing integrals like that in Eq. (16) diverges with the thickness of the film. However, below it will be seen that this divergence is removed by the proper normalization of the phonon eigenmodes $\left[F_{d}\right.$ and $F_{f}$ in Eqs. (11) and (12)] in the volume of the sample $V_{p}$ and the electron-phonon matrix element (13) remains finite.

\section{HEAT FLUX CARRIED BY LAMB MODES}

We can now calculate the power function $P$, i.e., the energy transferred from hot electrons to phonons in a unit of time:

$$
P=2 \sum_{\mathbf{k}_{\|}, \mathbf{q}_{\|}, \alpha, k_{z}, k_{z}^{\prime}} \hbar \omega_{\alpha}\left(\Gamma_{\alpha, k_{z}, k_{z}^{\prime}}^{\mathrm{em}}\left(\mathbf{k}_{\|} \rightarrow \mathbf{k}_{\|}-\mathbf{q}_{\| \mid}\right)-\Gamma_{\alpha, k_{z}, k_{z}^{\prime}}^{\mathrm{ab}}\left(\mathbf{k}_{\|} \rightarrow \mathbf{k}_{\|}+\mathbf{q}_{\|}\right)\right)
$$

The emission and absorption rates $\Gamma$ are given by the golden rule:

$$
\begin{gathered}
\Gamma^{\mathrm{em}}\left(\mathbf{k}_{\|} \rightarrow \mathbf{k}_{\|}-\mathbf{q}_{\|}\right)=\frac{2 \pi}{\hbar}\left|g_{\alpha, \mathbf{q}_{||}}^{k_{z}, k_{z}^{\prime}}\right|^{2}\left[n_{p}\left(\hbar \omega_{\alpha}\right)+1\right] \\
\times f\left(\epsilon_{\mathbf{k}_{||}, k_{z}}\right)\left[1-f\left(\epsilon_{\mathbf{k}_{||}-\mathbf{q}_{||}, k_{z}^{\prime}}\right)\right] \delta\left(\epsilon_{\mathbf{k}_{||}, k_{z}}-\epsilon_{\mathbf{k}_{||}-\mathbf{q}_{\|}, k_{z}^{\prime}}-\hbar \omega_{\alpha}\right),
\end{gathered}
$$

where electron $(e)$ and phonon $(p)$ indices identify the respective temperature in the Bose distribution function $n_{p, e}\left(\hbar \omega_{\alpha}\right)=\left\{\exp \left(\beta_{p, e} \hbar \omega_{\alpha}\right)-1\right\}^{-1}$ with $\beta_{p, e}=1 / k_{B} T_{p, e}$; in the Fermi distribution function the chemical potential is replaced by the Fermi energy for the considered low-temperature regime $f\left(\epsilon_{\mathbf{k}}\right)=\left\{\exp \left[\beta_{e}\left(\epsilon_{\mathbf{k}}-E_{F}\right)\right]+1\right\}^{-1}$. The phonon absorption part of (18),$-\Gamma_{\alpha}^{\mathrm{ab}}\left(\mathbf{k} \rightarrow \mathbf{k}+\mathbf{q}_{\|}\right)$, is obtained from the emission term $\Gamma_{\alpha}^{\mathrm{em}}\left(\mathbf{k} \rightarrow \mathbf{k}-\mathbf{q}_{\|}\right)$ by the space-time inversion $\left(\mathbf{q}_{\|} \longrightarrow-\mathbf{q}_{\|}\right.$and $\left.\omega_{\alpha} \longrightarrow-\omega_{\alpha}\right)$ using the identity for the Bose distribution $n(-y)+1=-n(y)$. The power function $P(T)$ can then be cast in the form of 
a difference between terms separately dependent on $T_{e}$ and $T_{p}$, as in Eq. (1), with the help of the following identity for the Fermi and Bose distribution functions:

$$
f(x)[1-f(x-y)]=n_{e}(y)[f(x-y)-f(x)] .
$$

Summation over momenta in Eq.(18) is replaced by integration in a standard way. In calculating the integrals one can then switch to electron density of states and carry out energy integration by using the identity:

$$
\int_{0}^{\infty}[f(x-y)-f(x)] d x=k_{B} T_{e} \ln \left(\frac{\exp \left(\beta_{e} y\right)+\exp \left(-\beta_{e} E_{F}\right)}{1+\exp \left(-\beta_{e} E_{F}\right)}\right)
$$

where the variable $y$ is defined by the phonon energy $y= \pm \hbar \omega_{\alpha}$ in the emission and absorption processes [see Eqs. (18)]. Taking into account that $\hbar \omega_{\alpha}, k_{B} T<<E_{F}$, the exponentially small terms in the round brackets on the right-hand-side of Eq. (21) can be neglected and we obtain the relation

$$
\int_{0}^{\infty}[f(x-y)-f(x)] d x \simeq y
$$

also used in [21]. However, a more physically transparent way is to note that at low temperatures the electron scattering takes place near the Fermi surface, i.e., $\hbar \omega_{\alpha}, k_{B} T<<\epsilon_{\mathbf{k}} \sim E_{F}$. This allows us to approximate the right-hand-side of Eq. (20) with $n_{e}(y) y \delta\left(\epsilon_{\mathbf{k}}-E_{F}\right)$, by using the known expression for the main term of the expansion $f(x-y)-f(x) \simeq-y \partial f / \partial x \simeq$ $y \delta\left(\epsilon_{\mathbf{k}}-E_{F}\right)$ [see, e.g., Eq. (5.42) in [35]]. It can also be seen that this approximation reproduces the result in Eq. (22); recall that $x=\epsilon_{k}$. Then the above-mentioned form of Eq. (18) is easily obtained as follows:

$$
P=P_{0}\left(T_{e}\right)-P_{0}\left(T_{p}\right)
$$

Here

$$
\begin{aligned}
P_{0}\left(T_{e}\right) & =\sum_{\mathbf{k}_{||}, k_{z}, k_{z}^{\prime}, \mathbf{q}_{||}, \alpha=d, f} \frac{32 \pi \hbar^{2} E_{F}^{2}}{9 \rho A} \omega_{\alpha}\left|F_{\alpha}\right|^{2}\left[q_{t} q\left(q_{l}^{2}+q^{2}\right)\right]^{2} n\left(\hbar \omega_{\alpha} / k_{B} T_{e}\right) \delta\left(\epsilon_{\mathbf{k}}-E_{F}\right) \\
& \times\left\{\begin{array}{l}
\cos ^{2}\left(L q_{t} / 2\right), \alpha=d \\
\sin ^{2}\left(L q_{t} / 2\right), \alpha=f
\end{array}\right\} S\left(k_{z}, k_{z}^{\prime}, \alpha, q_{l}\right) \delta\left(\epsilon_{\mathbf{k}_{||}, k_{z}}-\epsilon_{\mathbf{k}_{\|}-\mathbf{q}_{\| \mid}, k_{z}^{\prime}}-\hbar \omega_{\alpha}\right) .
\end{aligned}
$$

Equation (24) shows that in this leading order of the low-temperature expansion phonons are emitted and absorbed by electrons on the Fermi surface. 
Let us now consider the energy conservation condition imposed by the last $\delta$ function in Eq. (24) on the cosine of the angle between the electron and phonon wave vectors written in spherical coordinates $(\sin \theta \cos \varphi)$ :

$$
\delta\left(\frac{\hbar^{2}}{m}\left(k_{F} q \sin \theta \cos \varphi\right)+\frac{\hbar^{2}}{2 m}\left(k_{z}^{2}-\left(k_{z}^{\prime}\right)^{2}-q^{2}\right)-\hbar \omega_{\alpha}\right) .
$$

For the dilatational mode $(\alpha=d)$ we have $k_{z}^{\prime}=k_{z}$ from Eq. (15) and then from Eq. (24) we obtain

$$
\begin{aligned}
& P_{0}^{F G, d}\left(T_{e}\right)= \frac{32 \pi \hbar^{2} E_{F}^{2}}{9 \rho A} \frac{A d}{(2 \pi)^{3}} \int_{0}^{\infty} k^{2} d k \int_{0}^{\pi} \sin \theta d \theta \int_{0}^{2 \pi} d \phi \frac{A}{(2 \pi)^{2}}\left(\int_{0}^{\infty} \int_{0}^{2 \pi} q d q d \phi_{d}\right) \\
& \times \omega_{d}\left|F_{d}\right|^{2}\left[q_{t} q\left(q_{l}^{2}+q^{2}\right)\right]^{2} n_{e}\left(\hbar \omega_{d}\right) \delta\left(\frac{\hbar^{2} k^{2}}{2 m}-E_{F}\right) \cos ^{2}\left(L q_{t} / 2\right) \\
& \times \frac{m}{\hbar^{2} k q} \delta\left(\sin \theta \cos \phi-\frac{q}{2 k}-\frac{m \omega_{d}(q)}{\hbar k q}\right) .
\end{aligned}
$$

One can see that due to conservation of the $z$ component of the electron momentum the phonon is actually emitted in the direction orthogonal to $\mathbf{k}$, i.e., $|\sin \theta \cos \varphi|<<1$. Indeed, we can estimate $m \omega_{d} / \hbar k_{F} q \sim c_{t} / v_{F}<<1$ and, since $k_{F} \sim \pi / a_{0}$ ( $a_{0}$ is the lattice spacing), also $q / 2 k_{F}<<1$. So, the $\mathbf{k}$ vector is allowed to rotate without restrictions in the plane orthogonal to $\mathbf{q}_{\|}$, while $\mathbf{q}_{\|}$in its turn is free to rotate in the plane of the film. Thus, integration over the electron $(\theta, \phi)$ and phonon $\left(\phi_{d}\right)$ angles in Eq. (26) results in the multiplier $(2 \pi)^{2}$ (formal derivation is somewhat lengthier and leads to the same conclusion). Integration over the length of $\mathbf{k}$ is trivial and can also be expressed in terms of the electron density of states for the parabolic dispersion, $N\left(E_{F}\right)=A d m k_{F} /(\hbar \pi)^{2}$. We finally obtain the following expression for the $d$ mode:

$$
P_{0, d}\left(T_{e}\right)=\frac{32 \pi m^{2} E_{F}^{2}}{9 \rho \hbar^{2}} \frac{A d}{(2 \pi)^{3}} \int_{0}^{\infty} \omega_{d}\left|F_{d}\right|^{2}\left[q_{t} q\left(q_{l}^{2}+q^{2}\right)\right]^{2} n_{e}\left(\hbar \omega_{d}\right) \cos ^{2}\left(L q_{t} / 2\right) d q
$$

which after substitution of the expressions in Eq. (11) into Eq. (27) results in

$$
P_{0, d}\left(T_{e}\right)=\frac{\zeta(4)}{12 \pi^{2}} \frac{V_{e}\left(k_{B} T_{e}\right)^{4} k_{F}^{4} J^{2}}{\rho L \hbar^{2} c_{t}^{3}(1-J)^{3 / 2}}
$$

Calculation of the heat current due to the flexural phonon modes is more involved since $k_{z}$ is not conserved even in the long-wavelength approximation and can significantly differ from $k_{z}^{\prime}$, Eq. (16). It is then convenient to express the power function (24) in the cylindrical coordinates: 


$$
\begin{aligned}
P_{0, f}\left(T_{e}\right) & =\frac{16 E_{F}^{2}}{9 \rho} \frac{A d^{4}}{(2 \pi)^{4}}\left(\frac{m}{\hbar}\right)^{2} \\
& \times \int_{0}^{\infty} d q I(q)\left|p_{l}\right|^{2} \omega_{\alpha}\left|F_{\alpha}\right|^{2}\left[q_{t} q\left(q_{l}^{2}+q^{2}\right)\right]^{2} n\left(\hbar \omega_{q} / k_{B} T_{e}\right) \sin ^{2}\left(L q_{t} / 2\right)
\end{aligned}
$$

Here

$$
\begin{aligned}
I(q) & =\int_{0}^{k_{F}} \frac{d k_{\|}}{\sqrt{k_{F}^{2}-k_{\|}^{2}}} \int_{-k_{F}}^{k_{F}} d k_{z} \int_{-\infty}^{\infty} d k_{z}^{\prime} \\
& \times\left[\delta\left(k_{z}-\sqrt{k_{F}^{2}-k_{\|}^{2}}\right)+\delta\left(k_{z}+\sqrt{k_{F}^{2}-k_{\|}^{2}}\right)\right]\left|\int_{\sigma / 2-1}^{\sigma / 2} \exp \left(i d\left(k_{z}-k_{z}^{\prime}\right) z\right) z d z\right|^{2} \\
& \times \int_{0}^{2 \pi} d \phi \delta\left(\cos \phi+\frac{\left(k_{z}^{2}-\left(k_{z}^{\prime}\right)^{2}-q^{2}\right) / 2}{k_{\|} q}-\frac{m \omega_{f}}{\hbar k_{\|} q}\right)
\end{aligned}
$$

In (30) we have introduced the ratio $\sigma=L / d$ with the limit value $\sigma=1$ corresponding to the absence of the insulating membrane, i.e., to a purely metallic sample. The multiple integral $I(q)$ is calculated analytically in the Appendix and its substitution into Eq. (29), together with the solution (12) for the flexural mode, leads to the following expression:

$$
\begin{aligned}
& P_{0, f}\left(T_{e}\right)=\frac{V_{e}\left(k_{B} T_{e}\right)^{4} d^{3} m^{2} J^{2} E_{F}^{2}}{4 \sqrt{3} \pi^{3} \rho L^{4} c_{t}^{3} \hbar^{6}(1-J)^{3 / 2}}\left[(\sigma-1)^{2} \int_{0}^{\infty} x^{3}(-\ln x) n(x) d x\right. \\
& \left.\quad+\left(\frac{1}{2}+(\sigma-1)^{2} \ln \left(25.53 \frac{\hbar L c_{t} \sqrt{(1-J) / 3}}{d^{2} k_{B} T_{e}}\right)\right) \int_{0}^{\infty} x^{3} n(x) d x\right] .
\end{aligned}
$$

From Eq. (31) we obtain the final result for the contribution of the flexural modes:

$$
P_{0, f}\left(T_{e}\right)=\frac{0.0075 V_{e}\left(k_{B} T_{e}\right)^{4} k_{F}^{4} J^{2}}{\rho L \sigma^{3} \hbar^{2} c_{t}^{3}(1-J)^{3 / 2}}\left((\sigma-1)^{2} \ln \left[4.4 \frac{\sigma^{2} \hbar c_{t} \sqrt{1-J}}{L k_{B} T_{e}}\right]+\frac{1}{2}\right) .
$$

The argument in the square brackets is proportional to $T^{*} / T_{e} \sim \hbar c /\left(L k_{B} T_{e}\right)$, so that the $\log$ term is positive for temperature in the interval below the crossover, i.e., where the description of the phonon subsystem in terms of quasi-two-dimensional phonon confinement is applicable. Note also that $P_{0, f}\left(T_{e}\right)$ does not vanish even for a purely metallic slab $\left(V_{e}=V_{p}\right.$ and $\sigma=1$ ) when electrons experience an antisymmetric field created by the flexural vibration mode [Eq. (16)] and the respective overlap function vanishes $S\left(k_{z}=k_{z}^{\prime}, f, q_{l}=i p_{l}\right)=0$. This result demonstrates the point made in the Introduction that the non-zero contribution of the flexural modes to the heat transfer is due to scattering processes with $k_{z}^{\prime} \neq k_{z}$. One 
can also see that the above result scales with the surface of the sample $(P \sim A)$ for a free metallic film $(\sigma=1)$, but for a composite structure $(\sigma>1)$ the geometry dependence becomes more complicated even for an acoustically uniform medium.

To simplify the discussion we assume that the phonon temperature is much lower than $T_{e}$, so that the total density of the heat transfer power $Q$ is obtained as the sum of just the two contributions in Eqs. (28) and (32):

$$
Q=\left(P_{0, d}\left(T_{e}\right)+P_{0, f}\left(T_{e}\right)\right) / V_{e}
$$

Thus, for the considered case of an acoustically uniform metal-insulator composite slab we obtain the following expression:

$$
Q=\frac{0.0075\left(k_{B} T_{e}\right)^{4} k_{F}^{4} J^{2}}{\rho L \sigma^{3} \hbar^{2} c_{t}^{3}(1-J)^{3 / 2}}\left(1.2 \sigma^{3}+\frac{1}{2}+(\sigma-1)^{2} \ln \left(4.4 \frac{\hbar \sigma^{2} c_{t} \sqrt{1-J}}{L k_{B} T_{e}}\right)\right) .
$$

Its generalization to the case of two acoustically inequivalent rigidly bonded layers is much lengthier and will be presented in detail elsewhere. A typical example corresponds to a $\mathrm{Cu}$ film deposited on a silicon-nitride insulating membrane. Following the guidelines of the standard description of layered elastic media (see, e.g., [25, 26]), one can obtain the analytic solutions for the acoustic branches of the vibrational modes and respective normalization factors in the long-wavelength approximation. The result is that solutions of the Stoneley type (i.e., interface guided waves with the amplitude decreasing away from the interface) do not appear in the long-wavelength limit and it is well justified to keep the two types of modes considered above solely responsible for the low-temperature behavior also in this case.

We identify the material parameters corresponding to $\mathrm{Cu}$ and silicon nitride by the respective indices, $i=1$ and 2 . The mass of the composite slab is $M$ and the ratio $M / A$ replaces the product $\rho L$ in Eq. (34). Then, with the additional notations

$$
\begin{gathered}
R_{i}=\rho_{i} c_{t, i}^{2}\left(1-J_{i}\right), \quad \chi=\frac{1}{2}\left(\frac{R_{2}(L-d)^{2}-R_{1} d^{2}}{R_{2}(L-d)+R_{1} d}\right), \\
G=\frac{2 \hbar}{d^{2}} \sqrt{\frac{\left(R_{1} d^{3}+R_{2}(L-d)^{3}\right) / 3-\chi^{2}\left(R_{1} d+R_{2}(L-d)\right)}{\rho_{1} d+\rho_{2}(L-d)}},
\end{gathered}
$$

the long-wavelength dispersion of the dilatational acoustic mode can be written as

$$
\omega_{d}=2 q \sqrt{\frac{R_{1} d+R_{2}(L-d)}{\rho_{1} d+\rho_{2}(L-d)}} .
$$


Equation (36) reproduces the known result [32]. For the flexural mode we find:

$$
\omega_{f}=2 q^{2} \sqrt{\frac{\left(R_{1} d^{3}+R_{2}(L-d)^{3}\right) / 3-\chi^{2}\left(R_{2}(L-d)+R_{1} d\right)}{\rho_{1} d+\rho_{2}(L-d)}} .
$$

It is easy to check that these expressions correctly reproduce the limit of the acoustically uniform medium and amount to an effective renormalization of the parameters in Eq. (34) without qualitatively changing the temperature dependence. Indeed, by carrying out the calculations within the lines described in the previous case we obtain the following generalization of Eqs. (31) $-(\underline{33})$ for the power density function $Q\left(T_{e}\right)=Q_{d}\left(T_{e}\right)+Q_{f}\left(T_{e}\right)$, where

$$
\begin{gathered}
Q_{d}=\left(k_{B} T_{e}\right)^{4} \frac{\zeta(4)}{12 \pi^{2}} \frac{A k_{F}^{4} J_{1}^{2}}{\hbar^{2} M}\left(\frac{\rho_{1} d+\rho_{2}(L-d)}{R_{1} d+R_{2}(L-d)}\right)^{3 / 2}, \\
Q_{f}=\frac{\left(\kappa_{B} T_{e}\right)^{4}}{\pi^{3} 3^{2} 2^{7}} \frac{A k_{F}^{4} J_{1}^{2}}{\hbar^{2} M}\left((2 \chi / d+1)^{2}\left(\ln \left(G / k_{B} T_{e}\right)+13.3\right)+3.247\right) \\
\times\left(\frac{d^{2}\left(\rho_{1} d+\rho_{2}(L-d)\right)}{\left(R_{1} d^{3}+R_{2}(L-d)^{3}\right) / 3-\chi^{2}\left(R_{2}(L-d)+R_{1} d\right)}\right)^{3 / 2} .
\end{gathered}
$$

Figure 2 shows the relative contribution of the two modes as given by Eqs. (38) and (39) to the electron-phonon heat transfer $\left(Q_{f} / Q_{d}\right)$ for the two samples $M 1$ and $M 3$ which have been interpreted in Refs. [12, 13] as demonstrating a crossover in the sub-Kelvin region. The material parameters can be found in [12, 13, 15]: $\rho_{1}=8940 \mathrm{~kg} / \mathrm{m}^{3}, \rho_{2}=3290 \mathrm{~kg} / \mathrm{m}^{3}, c_{t, 1}=$ $2575 \mathrm{~m} / \mathrm{s}, c_{t, 2}=6200 \mathrm{~m} / \mathrm{s}, J_{1}=0.27, J_{2}=0.36, k_{F}=1.65 \times 10^{10} \mathrm{~m}^{-1}, L=30 \mathrm{~nm}, A=600 \times$ $300(\mu m)^{2}, d(M 1)=14 n m, d(M 3)=19 n m$, to a good approximation $M / A \simeq \rho_{2}(L-d)$ by taking into account that the surface of the $\mathrm{Cu}$ film in these experiments was smaller than the supporting membrane.

We can see that the contribution of the flexural mode to the heat transfer is comparable to the dilatational one and gains more "weight" towards lower temperatures due to the presence of the log term in Eq. (39). Moreover, Fig.2 indicates that the sample with a higher thickness ratio between the metal film and the insulating membrane (i.e., a smaller geometric factor: $\sigma(M 3)=49 / 19<\sigma(M 1)=44 / 14)$ has also a higher value of the power ratio $Q_{f} / Q_{d}$. Note also that the ratio $Q_{f} / Q_{d}$ reduces to a constant $(\simeq 0.41)$ for the case of a suspended metallic film, as can be easily seen from the Eqs. (28) and (32) with $\sigma=1$.

In Fig. 3 our result is compared to the temperature dependence of the total power density $Q=Q_{f}+Q_{d}$ for the sample $M 1$ (Fig. 3 in [12]) with the material parameters as given 




FIG. 2: The relative contribution $Q_{f} / Q_{d}$, Eqs. (38) and (39), to the heat flux of the flexural versus dilatational acoustic modes for the two samples $M 1$ and $M 3$ in [12, 13] (see text).

above. The effective value of $k_{F}$ is obtained by fitting the low-temperature region of $Q(T)$ and is slightly larger than $1.4 \times 10^{10} \mathrm{~m}^{-1}$ following from the known value of the Fermi energy for copper, $7 \mathrm{eV}$, if the effective mass $m$ is estimated from the electron heat capacity (e.g., [36]) for the simple isotropic parabolic dispersion. We mention that in [15] the deviation of the Fermi surface in noble metals from a simple spherical shape has been studied in terms of surface averaged effective electronic parameters and for $\mathrm{Cu}$ the estimated increase of the respective prefactor in the electron-phonon power function is comparable to our result. At temperatures above $250 \mathrm{mK}$ the analytical curve starts to deviate from the experiment as the crossover temperature is approached and higher-energy branches of the Lamb modes spectrum should be taken into account. From the Eqs.(38) and (39) it also follows that the M3 sample (not shown in Fig.(3)) has a somewhat higher value of the total power density $Q$ than $M 1$ for the considered temperature region. The quantitative comparison shows a good agreement with the results presented in Fig. 4 of [12] and in Fig. 3 of [13].

\section{CONCLUSIONS}

We have obtained explicit expressions for the power density of the electron-phonon heat transfer in a metallic film including the case when the film is deposited on an insulating membrane with generally different acoustic characteristics, Eqs. (38) and (39). The temperature 


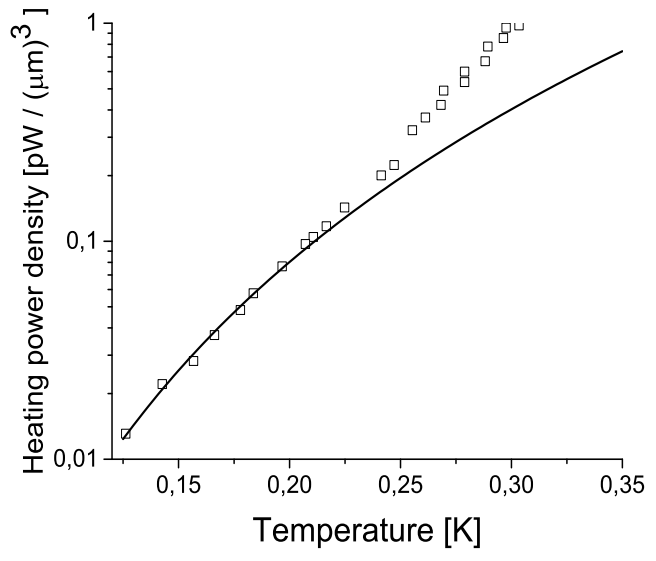

FIG. 3: The power density of the electron-phonon heat transfer for the $M 1$ sample in [12] (squares) and the joint contribution due to flexural and dilatational acoustical Lamb modes $Q=Q_{f}+Q_{d}$ as given by Eqs. (38) and (39) (see text).

regime covered by the present analysis corresponds to low temperatures when the phonon spectrum is dominated by quasi-two-dimensional modes of vibration. The long-wavelength approximation which considers only the lowest phonon branches is well justified at lower temperatures and has allowed us to carry out the calculations analytically. Thus, for the specific example considered above, e.g., Fig. 3, this description corresponds to temperatures below $0.25 \mathrm{~K}$ while the quasi-two-dimensional regime sets in around 0.4-0.5 K [12]. It turns out that the contributions of the flexural and dilatational phonon modes to the heat flux are of the same order of magnitude, as illustrated by Fig. 2 .

For a suspended metallic film, $\sigma=1$, the heat current follows a $T^{4}$ dependence, i.e., $P=\Sigma_{2 D} A\left(T_{e}^{4}-T_{p}^{4}\right)$, which appears to "fit the pattern" of the dimensionality dependence including the integer power $x$ in the $T^{x}$ temperature variation, namely, when the phonon subsystem corresponds to either a three-dimensional bulk material with the power index $x=5$ as in Eq. (1), or to a two-dimensional or quasi-two-dimensional material such as a single or bilayer graphene [37, 38] with $x=4$, or to a quasi-one-dimesnional nanowire [17] with $x=3$. However, this result could seem surprising in the context of previous works on some other quasi-two-dimensional systems, e.g., [20, 21], for the case of a semiconductor quantum well, which would rather suggest a fractional value of $x$ due to the peculiar quadratic dispersion of the flexural mode. Fractional power, $T^{2.5} \ln T$, was also reported for 
the flexural modes' contribution to electrical resistivity in free-standing graphene [39]. The value $x=4$ for the heat flux in graphene is actually due to electron coupling with the dilatational phonons, since the "troublesome" flexural ones couple to electrons only in second order in the displacement and can be disregarded for the graphene on a substrate as well [37]. Moreover, essentially different contributions of the phonon modes could be expected to result not only from the linearity and non-linearity of the respective dispersion laws, but also from the explicit presence of the size (thickness $L$ ) dependence in the dispersion of the flexural mode [e.g., compare $\omega_{d}$ and $\omega_{f}$ in Eqs. (11) and (12)]. Indeed, in the studies on phonon transport and phonon heat capacity of a free-standing dielectric membrane [40, 41] a striking difference between the thickness dependent and non-dependent behavior has been derived for the two types of vibration. In contrast, for the electron-phonon heat transfer we find a comparable contribution for the two modes even when the metallic film is deposited on an insulating membrane $(\sigma>1)$, when the effect of sample geometry on the power $P$ is more complicated than a simple scaling with surface area $A$ [see, e.g., Eqs. (28) and (32) for the acoustically uniform sample]. As we have shown such unexpected relative similarity in the temperature and size (e.g., thickness) dependence results from several physical factors, so that their combination differs from the cases studied earlier. For a coupled electron-phonon system both the dispersions and the amplitudes of the excitations play an important role. Respectively, a proper normalization of the amplitudes is crucial. One can then see, e.g., from Eqs. (11) and (12), that the normalization of the phonon modes in Eqs. (6) and (17), containing the multipliers $F_{d, f}$ and the auxiliary parameters $q_{t, l}$, moderates to some extent the sharp differences between the $d$ and $f$ dispersions. It is also clear that this "compensation" depends on specific quantity considered, which in our case is the heat flux. Unlike the case of graphene or that of a semiconductor quantum well, the electron excitations are described in terms of three-dimensional Fermi gas with parabolic dispersion. Respectively, the overlap of the electronic amplitudes in the initial and scattered states [Eq. (14)] is also a physical factor which differs from the models considered before. As discussed in the text, this overlap strongly discriminates between the $d$ and $f$ phonons and contributes to the above-mentioned "compensation" as well.

For the case of a metallic film deposited on an insulating membrane the flexural mode contribution to the power density of the electron-phonon heat transfer acquires an additional logarithmic term of the form $T^{4} \ln T$ [Eq. (39)], while that of dilatational modes keeps the 
$T^{4}$ dependence [Eq. (38)]. As we have shown, such functional dependence reproduces well the observed behavior, which was modeled in [12] with a power law $T^{x}$ with $x<$ 4.5. The dependence on the material parameters for a metallic film with dielectric backing becomes more complicated, especially for the acoustically non-uniform case. This point can be illustrated by a more detailed analysis of the experimental work cited above. Thus,

one generally expects that reducing the dimensionality would enhance the electron-phonon heat exchange. This is confirmed by Figs. 2 and 4 in [12] for sufficiently thick samples to be considered bulklike (e.g., $M 5$ and $B 1$ ) and which show a much lower power density $Q\left(=P / V_{e}\right)$ than the thinner samples (like $M 1$ and $M 3$ discussed earlier, with $M 3$ being thicker than $M 1)$. Moreover, if the simple surface area scaling of the power function $(P \sim A)$ would be valid for these thinner samples, then one would expect that $Q(M 1)>Q(M 3)$. However, the power density for the thicker of the two (M3) is larger than for the thinner one $(M 1)$ in the considered temperature region. This non-trivial dependence on the material parameters is well reproduced by the analytical expressions. Thus, the present analysis demonstrates that in thin metal films both types of Lamb's phonon modes should be taken into account on equal footing for a better understanding of the electron-phonon heat transfer at low temperatures.

\section{Acknowledgments}

This work has been financially supported by UEFISCDI (Romania), Project No. IdeiPCE 114/2011, and by ANCS, Project No. PN09370102.

\section{APPENDIX}

Integration over the angle $\phi$ and over $k_{z}$ in (30) reduces to a tripple integral

$$
\begin{aligned}
& I(q)=\int_{0}^{k_{F}} \frac{d k_{\|}}{\sqrt{k_{F}^{2}-k_{\|}^{2}}} \int_{-\infty}^{\infty} d k_{z}^{\prime} \frac{\theta\left(1-\left(\left[k_{F}^{2}-k_{\|}^{2}-\left(k_{z}^{\prime}\right)^{2}\right] / 2 k_{\|} q-D q / k_{\|}\right)^{2}\right)}{\sqrt{1-\left(\left[k_{F}^{2}-k_{\|}^{2}-\left(k_{z}^{\prime}\right)^{2}\right] / 2 k_{\|} q-D q / k_{\|}\right)^{2}}} \\
& \left(\left|\int_{\sigma / 2-1}^{\sigma / 2} \exp \left(i d\left(\sqrt{k_{F}^{2}-k_{\|}^{2}}-k_{z}^{\prime}\right) z\right) z d z\right|^{2}+\left|\int_{\sigma / 2-1}^{\sigma / 2} \exp \left(i d\left(\sqrt{k_{F}^{2}-k_{\|}^{2}}+k_{z}^{\prime}\right) z\right) z d z\right|^{2}\right),
\end{aligned}
$$


where $\theta(x)$ is the Heaviside function and we have introduced the constant

$$
D=1 / 2+m L c_{t} \sqrt{(1-J) / 3} / \hbar>0
$$

taking into account the dispersion of the flexural mode in (12).

We further use the dimensionless variables

$$
x=\sqrt{k_{F}^{2}-k_{\|}^{2}} / k_{F} ; \quad y=k_{z}^{\prime} / k_{F} ; \quad Q=q / k_{F} .
$$

Eq. (A1) then transforms into

$$
I(q)=2 q \int_{0}^{1} d x \int_{-\infty}^{\infty} d y \frac{\theta\left(4 Q^{2}\left(1-x^{2}\right)-\left(x^{2}-y^{2}-2 D Q^{2}\right)^{2}\right)}{\sqrt{4 Q^{2}\left(1-x^{2}\right)-\left(x^{2}-y^{2}-2 D Q^{2}\right)^{2}}}\left(G^{-}+G^{+}\right),
$$

where we have defined the functions

$$
G^{\mp} \equiv G(y \mp x)=\left|\int_{\sigma / 2-1}^{\sigma / 2} \exp \left(i d k_{F}(y \mp x) z\right) z d z\right|^{2} .
$$

We next consider the contribution $I^{-}(q)$ to $I(q)$ corresponding to $G^{-}$and switch to new variables

$$
u=y-x ; \quad v=x
$$

Then $I^{-}(q)$ takes the following form

$$
I^{-}=2 q \int_{-\infty}^{\infty} d u\left|\int_{\sigma / 2-1}^{\sigma / 2} \exp \left(i d k_{F} u z\right) z d z\right|^{2} \int_{0}^{1} \frac{\theta\left(c-b v-a v^{2}\right) d v}{\sqrt{c-b v-a v^{2}}}
$$

where $a, b$ and $c$ are functions of $u$ :

$$
\begin{gathered}
a=4\left(Q^{2}+u^{2}\right)>0, \\
b=4 u\left(u^{2}+2 D Q^{2}\right), \\
c=4 Q^{2}-\left(u^{2}+2 D Q^{2}\right)^{2} .
\end{gathered}
$$

Integration over $v$ gives

$$
\int_{0}^{1} \frac{\theta\left(c-b v-a v^{2}\right) d v}{\sqrt{c-b v-a v^{2}}}=\frac{1}{\sqrt{a}}\left(\frac{\pi}{2}-\arctan \left(\frac{b \theta(c)}{2 \sqrt{a c}}\right)\right) .
$$

Returning then to the definitions in (A4) and considering $G^{+}$we now define the variables $u$ and $v$ by changing the sign respectively: $u=y+x$ and $v=x$. This results in the expression 
for the $I^{+}(q)$ which differs from $I^{-}(q)$ in (A6) by the change of sign in front of $b$, so that integration over $v$ will differ from (A8) by the sign in front of the arctan function. These terms then cancel each other in Eq. (A3) when expressed in the $u$ and $v$ variables, so that our integral simplifies to

$$
I=\pi q \int_{-\infty}^{\infty}\left|\int_{\sigma / 2-1}^{\sigma / 2} \exp \left(i d k_{F} u z\right) z d z\right|^{2} \frac{d u}{\sqrt{Q^{2}+u^{2}}}
$$

The $z$ - integral can also be integrated exactly, so that after the replacement of variable $x=u d k_{F}$, Eq. (A9) becomes

$$
I=\int_{-\infty}^{\infty} \frac{2 \sigma(\sigma-2) x^{2}(1-\cos x)+4 x^{2}+8(1-\cos x-x \sin x)}{4 x^{4} \sqrt{(d q)^{2}+x^{2}}} d x
$$

The last integral can be reduced to the Meijer G-function [42], however for the long wavelength approximation $d q<1$ one can also obtain directly from (A10) its excellent approximation for $d q<1$ by the following expression

$$
I(q) \simeq \pi q\left(-\frac{(\sigma-1)^{2}}{2} \ln (d q)+0.81(\sigma-1)^{2}+\frac{1}{8}\right)
$$

[1] H. Q. Nguyen, M. Meschke, H. Courtois, and J. P. Pekola, Phys. Rev. Appl. 2, 054001 (2014).

[2] J. T. Muhonen, M. Meschke, and J. P. Pekola, Rep. Prog. Phys. 75, 046501 (2012).

[3] A. V. Feshchenko, L. Casparis, I. M. Khaymovich, D. Maradan, O.-P. Saira, M. Palma, M. Meschke, J. P. Pekola, and D. M. Zumbuhl, Phys. Rev. Appl. 4, 034001 (2015).

[4] J. Ziman, Electrons and Phonons: The Theory of Transport Phenomena in Solids (Clarendon, Oxford, 1960).

[5] M. I. Kaganov, I. M. Lifshitz, and L. V. Tanatarov, Zh. Eksp. Tear. Fiz. 31, 232 (1956) [Sov. Phys. JETP 4, 173 (1957).

[6] P.B. Allen, Phys. Rev. Lett. 59, 1460 (1987).

[7] F. C. Wellstood, C. Urbina, and John Clarke, Phys. Rev. B, 49, 5942 (1994).

[8] A. Sergeev and V. Mitin, Phys. Rev. B 61, 6041 (2000).

[9] J. T. Karvonen, L. J. Taskinen, and I. J. Maasilta, Phys. Rev. B 72, 012302 (2005).

[10] M. A. Stroscio and M. Dutta, Phonons in Nanostructures (CUP, United Kingdom, 2004). 
[11] A. N. Cleland, Foundations of Nanomechanics (Springer-Verlag Berlin Heidelberg, 2003).

[12] J. T. Karvonen and I. J. Maasilta, Phys. Rev. Lett. 99, 145503 (2007).

[13] J. Karvonen and I. Maasilta, J. Phys.: Conf. Series, 92, 012043 (2007).

[14] J. F. DiTusa, K. Lin, M. Park, M. S. Isaacson, and J. M. Parpia, Phys. Rev. Lett. 68, 1156 (1992).

[15] S.-X. Qu, A. N. Cleland, and M. R. Geller, Phys. Rev. B, 72, 224301 (2005).

[16] J. M. Underwood, P. J. Lowell, G. C. O’Neil, and J. N. Ullom, Phys. Rev. Lett. 107, 255504 (2011).

[17] F. W. J. Hekking, A. O. Niskanen, and J. P. Pekola, Phys. Rev. B, 77, 033401 (2008).

[18] J. T. Muhonen, A. O. Niskanen, M. Meschke, Yu. A. Pashkin, J. S. Tsai, L. Sainiemi, S. Franssila, and J. P. Pekola, Appl. Phys. Lett. 94, 073101 (2009).

[19] B. A. Auld, Acoustic Fields and Waves in Solids, 2nd Ed. (Robert E. Krieger Publishing Company, 1990).

[20] N. Bannov, V. Aristov, V. Mitin, and M.A. Stroscio, Phys. Rev. B, 51, 9930 (1995).

[21] B. A.Glavin , V. I.Pipa, V. V.Mitin and M. A. Stroscio, Phys. Rev. B 65, 205315 (2002).

[22] S.K. Mahatha and K.S.R. Menon, J. Electron Spectrosc. 193, 43 (2014).

[23] Z. Lindenfeld and R. Lifshitz, Phys. Rev. B 87, 085448 (2013).

[24] P. J. Koppinen and I. J. Maasilta, Phys. Rev. Lett. 102, 165502 (2009).

[25] L. M. Brekhovskikh, Waves in Layered Media (Academic Press, New York, 1980).

[26] J. L. Rose, Ultrasonic Waves in Solid Media (Cambridge University Press, UK, 2004).

[27] J. D. N. Cheeke, Fundamentals and Applications of Ultrasonic Waves (CRC Press LLC, USA, 2002).

[28] L. M. A. Pascal, A. Fay, C. B. Winkelmann, and H. Courtois, Phys. Rev. B 88, 100502(R) (2013).

[29] E. Swartz, and R. Pohl, R., Rev. Mod. Phys. 61, 605 (1989).

[30] F. Giazotto, T.T. Heikkilä, A. Luukanen, A. Savin, and J. P. Pekola Rev. Mod. Phys. 78, 217 (2006).

[31] V. J. Kauppila, H. Q. Nguyen, and T. T. Heikkilä, Phys. Rev. B 88, 075428 (2013).

[32] Wang Xiao-Min, Lian Guo-Xuan and Li Ming-Xuan, Chinese Phys. Lett. 20, 1084 (2003).

[33] D. V. Anghel, and T. Kühn, J. Phys. A: Math. Theor. 40, 10429 (2007)

[34] T. Kühn, and D. V. Anghel, Y. M. Galperin, and M. Manninen, Phys. Rev. B, 76, 165425 
(2007).

[35] G. Rickayzen, Green's Functions and Condensed Matter (Dover Publications, New York, 2013).

[36] D. L. Martin, Phys. Rev. B 8, 5357 (1973).

[37] J. K. Viljas and T. T. Heikkilä, Phys. Rev. B 81, 245404 (2010).

[38] S. S. Kubakaddi, Phys. Rev. B 79, 075417 (2009).

[39] E. Mariani and F. von Oppen, Phys. Rev. Lett. 100, 076801 (2008); 100, 249901(E) (2008).

[40] T. Kühn, D. V. Anghel, J. P. Pekola, M. Manninen, and Y. M. Galperin, Phys. Rev. B 70, 125425 (2004).

[41] O. V. Fefelov, J. Bergli, and Y. M. Galperin, Phys. Rev. B 75, 172101 (2007).

[42] NIST Handbook of Mathematical Functions, edited by F. W. J. Olver, D.W.Lozier, R.F.Boisvert, andC.W.Clark (Cambridge University Press, Cambridge, England, 2010). 